\title{
Structure and Formation Mechanism of the Transfermium Isotope ${ }^{254} \mathrm{No}$
}

\author{
P. Reiter ${ }^{1}$, T.L. Khoo ${ }^{1}$, C.J. Lister ${ }^{1}$, D. Seweryniak ${ }^{1}$, I. Ahmad ${ }^{1}$, \\ M. Alcorta ${ }^{1}$, M.P. Carpenter ${ }^{1}$, J.A. Cizewski ${ }^{1,3}$, C.N. Davids ${ }^{1}$, \\ G. Gervais ${ }^{1}$, J.P. Greene ${ }^{1}$, W.F. Henning ${ }^{1}$, R.V.F. Janssens ${ }^{1}$, \\ T. Lauritsen ${ }^{1}$, S. Siem ${ }^{1,8}$, A.A. Sonzogni ${ }^{1}$, D. Sullivan ${ }^{1}$, J. Uusitalo $^{1}$, \\ I. Wiedenhöver ${ }^{1}$, N. Amzal ${ }^{2}$, P.A. Butler ${ }^{2}$, A.J. Chewter ${ }^{2}$, K.Y. Ding ${ }^{3}$, \\ N. Fotiades ${ }^{3}$, J.D. Fox ${ }^{4}$, P.T. Greenlees ${ }^{2}$, R.-D. Herzberg ${ }^{2}$,

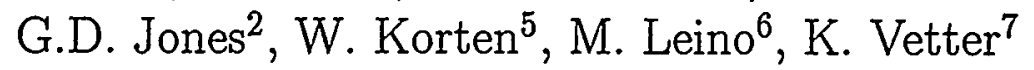

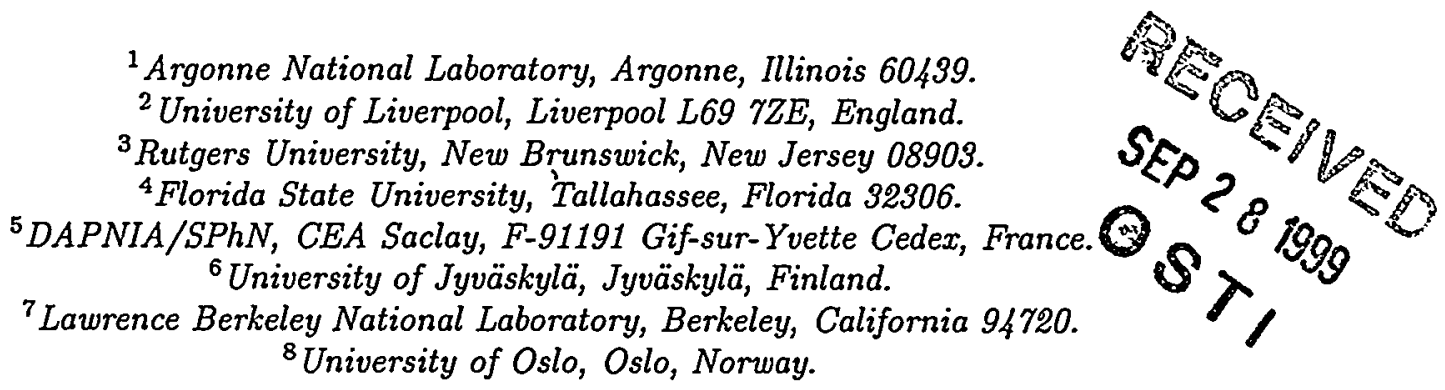

\begin{abstract}
The ground-state band of the $\mathrm{Z}=102$ isotope ${ }^{254}$ No has been identified up to spin 14, indicating that the nucleus is deformed. The deduced quadrupole deformation, $\beta=0.27$, is in agreement with theoretical predictions. These observations confirm that the shell-correction energy responsible for the stability of transfermium nuclei is partly derived from deformation. The survival of ${ }^{254}$ No up to spin 14 means that its fission barrier persists at least up to that spin.
\end{abstract}

\section{INTRODUCTION}

Experimental information about the formation and structure of heavy elements is of pivotal interest in the search for the long-predicted island of spherical superheavy nuclei. Despite a vanishing liquid drop fisşion barrier, the elements with $\mathrm{Z} \gtrsim 100$ are stable against fission. A large shell correction energy creates a fission barrier of up to $8 \mathrm{MeV}[1,2]$ and a 'peninsula' of stability has been found from $\mathrm{Z}=102$ to 112 , where nuclei decay preferentially by $\alpha$ emission [3]. For the predicted 'island' of superheavy nuclei at $Z=114, N=184$ the stability is based on doubly-closed spherical proton and neutron shells [4]. The shell correction energy of lighter transfermium 


\section{DISCLAIMER}

This report was prepared as an account of work sponsored by an agency of the United States Government. Neither the United States Government nor any agency thereof, nor any of their employees, make any warranty, express or implied, or assumes any legal liability or responsibility for the accuracy, completeness, or usefulness of any information, apparatus, product, or process disclosed, or represents that its use would not infringe privately owned rights. Reference herein to any specific commercial product, process, or service by trade name, trademark ${ }_{\bar{i}}$ manufacturer, or otherwise does not necessarily constitute or imply its endorsement, recommendation, or favoring by the United States Government or any agency thereof. The views and opinions of authors expressed herein do not necessarily state or reflect those of the United States Government or any agency thereof. 


\section{DISCLAIMER}

Portions of this document may be illegible in electronic image products. Images are produced from the best available original document. 
nuclei (TFN) is maximized for deformed shapes, with not only quadrupole, but also higher multipole moments being important around ${ }^{256}$ No [5]. However, hitherto there is no direct experimental confirmation of the deformation. The observation of the ground state band in at least one nucleus will provide important confirmation of the models, and provide a benchmark for extrapolation to heavier systems.

Observation of high spin states provides important information about the fission barrier at high angular momentum, which is intimately related to the formation mechanism of heavy elements via fusion-evaporation reactions. Theoretical predictions of the barrier and the shell correction energies are calculated for only the ground state of TFN. The barrier may decrease with increasing angular momentum and it is not obvious that high spin states will survive due to a high fissility.

\section{EXPERIMENTAL PROCEDURE}

The unprecedented sensitivity achieved by combining Gammasphere and the Argonne Fragment Mass Analyzer (FMA) makes it possible to study the nuclear structure of TFN with $(\mathrm{HI}, \mathrm{xn})$ reactions. The interesting reaction products are formed with cross sections in the $\mu \mathrm{b}$ region. Prompt $\gamma$ rays are required to be in coincidence with the very weakly produced evaporation residues, which have to be identified from $a>10^{4}$ more intense fission background in the residue separator. Moreover, Gammasphere is an efficient detector array with high granularity, which is suitable for determining the two-dimensional multiplicity (fold)/ sum-energy distribution of evaporation residues. From these observables, the entry spin and the excitation energy of the residues can be obtained.

A first experiment was performed to produce ${ }^{254}$ No via the ${ }^{208} \mathrm{~Pb}\left({ }^{48} \mathrm{Ca}, 2 \mathrm{n}\right)$ reaction at a bombarding energy of $215 \mathrm{MeV}$. Excitation function measurements [6] determined a production cross section of $\approx 3 \mu \mathrm{b}$ for the cold-fusion reaction at this beam energy. The target and projectile combination of two doubly-closed shell nuclei results in a large negative $Q$-value and hence, a low compound nucleus excitation energy $(19.3 \mathrm{MeV})$. As a consequence, the $2 \mathrm{n}$ evaporation channel is essentially the only open one, and charged particle evaporation is not observed [6].

The Argonne superconducting linear accelerator ATLAS provided ${ }^{48} \mathrm{Ca}$ beams of up to $9 \mathrm{pnA}$. The ${ }^{208} \mathrm{~Pb}$ targets $\left(500 \mu \mathrm{g} / \mathrm{cm}^{2}\right)$ were mounted on a rotating target wheel to prevent destruction by the beam. In addition, the beam was dispersed vertically by $\pm 2.5 \mathrm{~mm}$ through wobbling with a magnetic steerer.

Prompt $\gamma$ rays from ${ }^{254}$ No were detected with Gammasphere, consisting of 101 Compton suppressed Ge detectors. At the FMA focal plane, charged particles were detected using either a position-sensitive multi-channel plate detector or a parallelplate avalanche counter. After transmission through the focal-plane detector, the residues were implanted in a double-sided Si strip detector (DSSD), consisting of $16001 \times 1 \mathrm{~mm}$ pixels. 
,
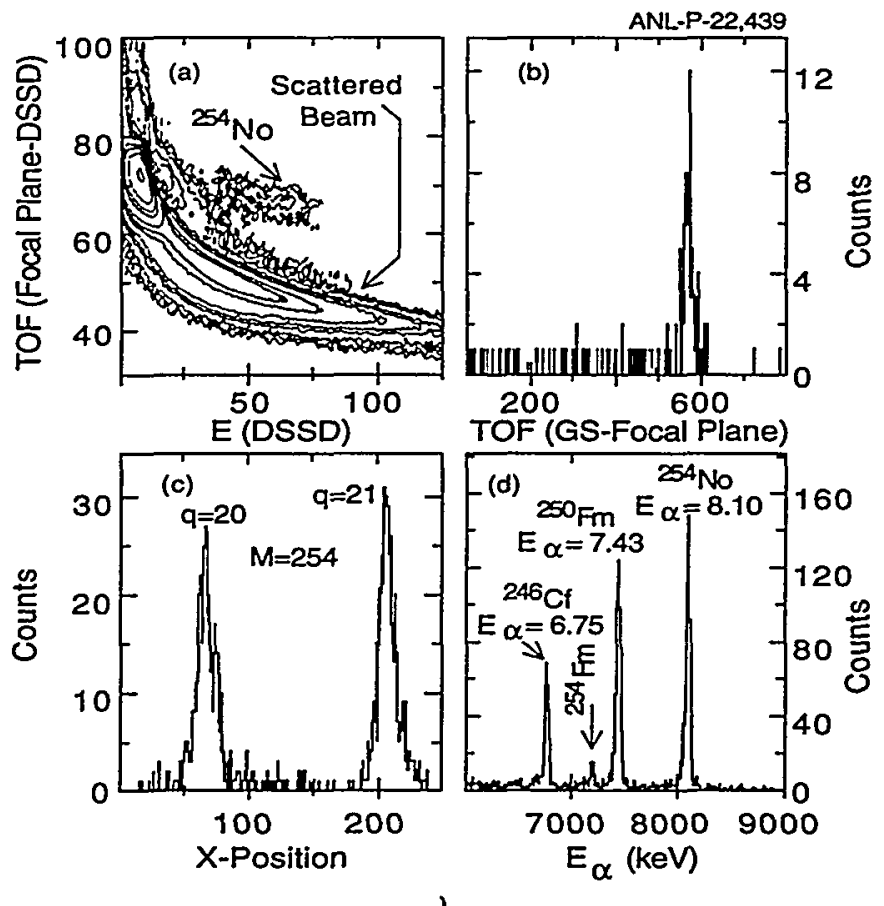

FIGURE 1. (a) Two-dimensional spectrum of the flight time from the focal-plane detector to the DSSD vs. the evaporation-residue implant energy in the DSSD. (b) Time-of-flight spectrum from Gammasphere to the focal-plane detector. (c) Mass/charge spectrum at the focal-plane detector. (d) Alpha spectrum from the DSSD detector, showing the peaks from three generations of $\alpha$ decay, starting with ${ }^{254} \mathrm{No}$. (The ${ }^{254} \mathrm{Fm} \alpha$ peak follows two successive electron-capture decays from ${ }^{254} \mathrm{No}$ ). In (a-c), the axes are in channel numbers.

\section{DATA ANALYSIS AND RESULTS}

\section{Ground State Band and Moments of Inertia}

To separate the nobelium residues from scattered beam particles and fission fragments, gates were set on the two-dimensional histogram of flight time between the focal-plane detector and the DSSD vs. DSSD implant energy (Fig. 1a). Coincidence gates were also required on: (i) the time of flight of the residues from the the target to the focal plane (Fig. 1b) and (ii) the focal-plane detector positions corresponding to two charge states ( $q=20$ and 21 ) of ${ }^{254}$ No (Fig. 1c). After implantation of a ${ }^{254}$ No evaporation residue in a specific pixel, subsequent $\alpha$-decay in that pixel is used to identify ${ }^{254} \mathrm{No}$, with additional requirements of energy $(8.093 \mathrm{MeV})$ and decay time (Fig. 1d). The measured ${ }^{254}$ No half-life $t_{1 / 2}=51 \pm 6 \mathrm{~s}$ agrees with the known 55 s half-life.

Correlations of $\gamma$ rays with a focal-plane signal corresponding to a properly identified No residue constitutes the recoil decay tagging (RDT) technique, whereby unambiguous identification of the $\gamma$-ray parentage is achieved in the spectrum shown 


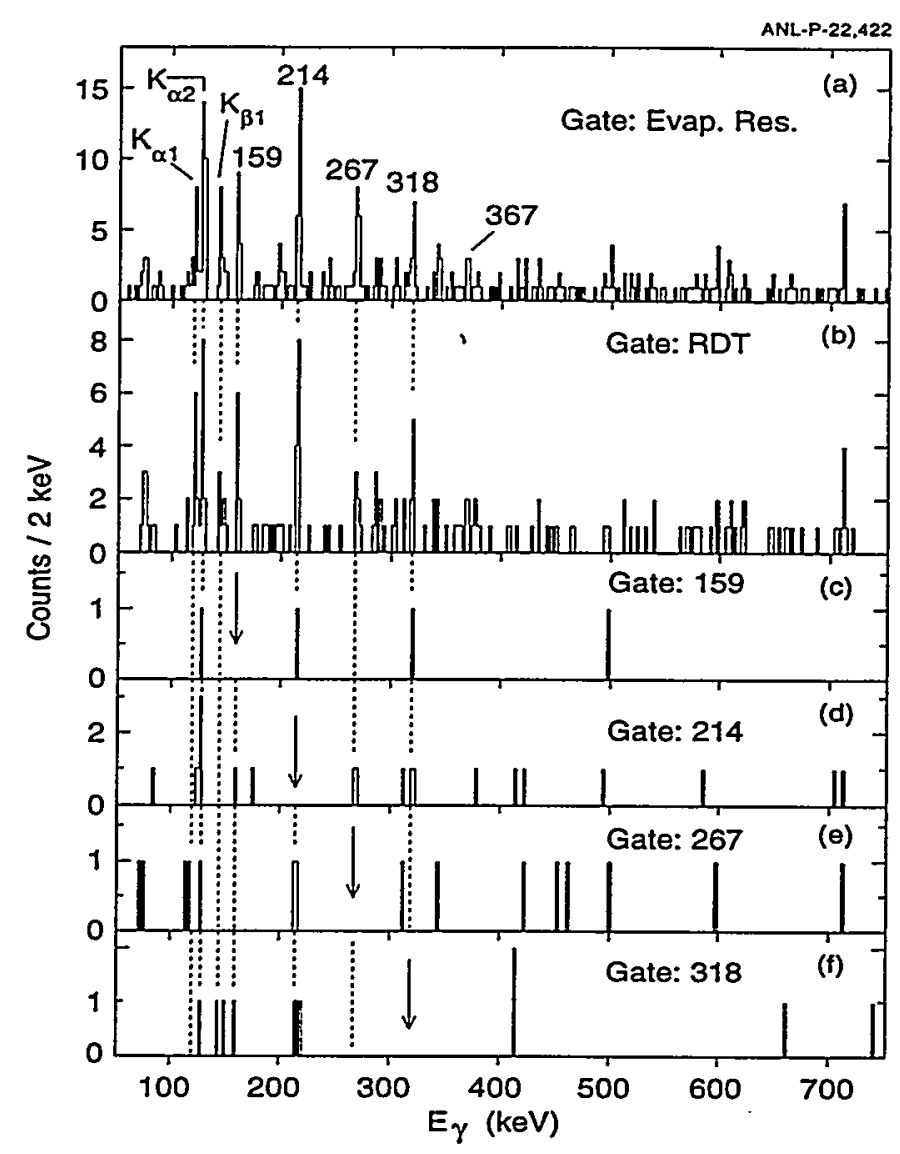

FIGURE 2. (a) Gamma spectrum obtained using coincidence gates on the 'peaks' in Fig. 1 (a-c). The background level is 0.4 counts/channel. Peaks labelled by energy are assigned as transitions within the ground-state band of ${ }^{254}{ }^{\prime}$ o. (b) Spectrum with an additional requirement on the ${ }^{254}$ No $\alpha$ peak shown in Fig. Id. (c-f) Coincidence spectra from gates set on the transitions indicated by arrows. Vertical dashed lines help visual alignment of peaks in the different panels.

in Fig. 2b. This spectrum affirms that the spectrum in Fig. 2a, which is generated without the additional RDT requirements, contains only ${ }^{254}$ No $\gamma$ rays. Coincidence gates on individual transitions, are given in Fig. 2 c-f. They support the assignment that the transitions labeled with energies constitute a cascade corresponding to the ground-state band of ${ }^{254}$ No.

The identification of a rotational band in ${ }^{254}$ No immediately establishes that the nucleus is deformed and confirms predictions of theories calculating shell-correction energies of TFN. Fig. 3 shows the moments of inertia, $\mathcal{J}^{(1)}$ and $\mathcal{J}^{(2)}$, for the groundstate band of ${ }^{254}$ No. $\left(\mathcal{J}^{(1)}=\hbar^{2}(2 I-1) / E_{\gamma}(I) ; \mathcal{J}^{(2)}=4 \hbar^{2} /\left(E_{\gamma}(I)-E_{\gamma}(I-2)\right)\right.$; $\hbar \omega=E_{\gamma} / 2$.) The Harris parameterization of the moments of inertia

$$
\mathcal{J}^{(1)}=\mathcal{J}_{0}+\mathcal{J}_{1} \omega^{2}, \quad \mathcal{J}^{(2)}=\mathcal{J}_{0}+3 \mathcal{J}_{1} \omega^{2}
$$

provides excellent fits of $\mathcal{J}^{(2)}(\omega)$ and $\mathcal{J}^{(1)}(\omega)$. From the parameters $\mathcal{J}_{0}$ and $\mathcal{J}_{1}$, we 


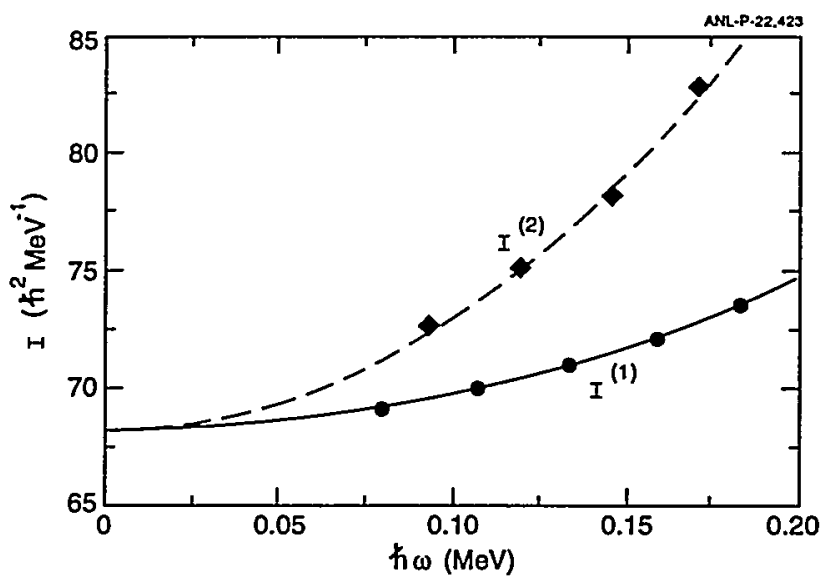

FIGURE 3. Moments of inertia, $\mathcal{J}^{(1)}$ and $\mathcal{J}^{(2)}$, for the ground state band of ${ }^{254}$ No. The lines are fits to the data, using $\mathcal{J}_{0}=68.2 \hbar^{2} \mathrm{MeV}^{-1}, \mathcal{J}_{1}=164.9 \hbar^{2} \mathrm{MeV}^{-1}$.

deduced the spins of the emitting states, using a procedure described in Ref. [7] and the expression

$$
I=\mathcal{J}_{0} \omega+\mathcal{J}_{1} \omega^{3}+1 / 2
$$

The spins have even integer values between 6 and 14 (within .01), providing support for assigning the transitions to the ground-state band.

The proposed level scheme of ${ }^{254} \mathrm{No}$ is shown in Fig. 4. We estimate the energies of transitions from the $2^{+}$and $4^{+}$states as $44(1)$ and $102(1) \mathrm{keV}$, respectively. These $\gamma$ rays were not detected because the states decay almost entirely by internal electron conversion. The deduced transition energies also conform to those extrapolated from neighboring lighter nuclei, providing additional support for the assigned spins.

\section{Sum-energy/Multiplicity Distribution}

The analysis of the multiplicity vs. sum-energy distribution (Fig. 5) suggests that residues are formed with spins up to $\sim 18 \hbar$. At $\mathrm{E}_{L_{a b}}=215 \mathrm{MeV}$ the ${ }^{208} \mathrm{~Pb}\left({ }^{48} \mathrm{Ca}, 2 \mathrm{n}\right)$ cold-fusion reaction can populate excited states in a limited range above the (measured) yrast line, up to the maximum excitation energy $\mathrm{E}^{\max }=E_{C N}-S_{n 1}-S_{n 2}$, after emission of two neutrons. The phase space is further limited by the kinetic energy of the neutrons. The populated states after neutron emission would lie below the yrast line for spins larger than 22. A new measurement at higher beam energies, leading to higher excitation energies, would bring larger spins into the No residue, so that the angular momentum dependence of the fission barrier can be studied and higher-spin states of the ground-state band perhaps identified. 


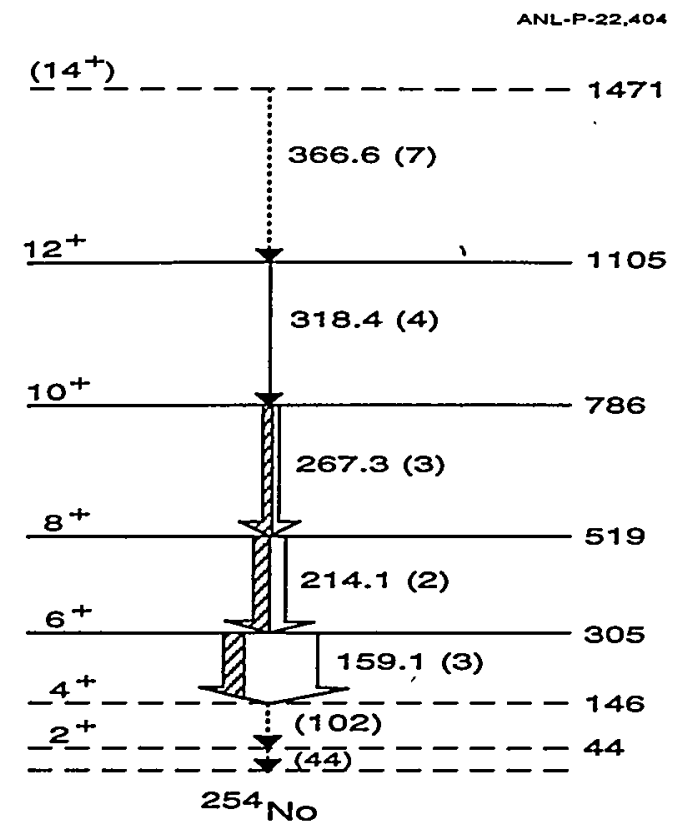

FIGURE 4. Proposed level scheme for the ground-state band of ${ }^{254}$ No. Spins are deduced using Eq. 3; the parity is assumed to be positive. The energies of the lowest two transitions, which were not detected decay because they decay by internal conversion electrons, were deduced by extrapolation. The $14^{+} \rightarrow 12^{+}$assignment is tentative. The widths of the filled and open arrows are proportional to the $\gamma$ and electron intensities, respectively; the latter were computed by assuming that the transitions have E2 multipolarity. Note the large conversion coefficients in nobelium. The transition intensities decrease as spin grows, as expected for a $(\mathrm{HI}, \mathrm{xn})$ reaction.

\section{DISCUSSION AND CONCLUSION}

The $\mathrm{B}(\mathrm{E} 2)$ values of rotors are related to the $2^{+}$level energies by empirical formulae [8-10]. By using Eq. 4 of Ref. [9] and equations from Ref. [10] relating the $\mathrm{B}(\mathrm{E} 2)$, quadrupole moment and deformation, we deduce a quadrupole deformation parameter of $\beta=0.27(2)$ for ${ }^{254} \mathrm{No}$. (The uncertainty is given by the systematic deviations between measured $\mathrm{B}(\mathrm{E} 2)$ values in heavy nuclei and those deduced from the empirical relationship of Ref. [9].)

This value is in agreement with a value of 0.25 given by different macroscopicmicroscopic model calculations [5,11,12], and with respective values of 0.27 and 0.26 from a Hartree-Fock-Bogoliubov (HFB) calculation with the SLy4 force [13] and from a relativistic Hartree-Bogoliubov calculation with the NL3 Lagrangian parameterization [14]. Other HFB and relativistic mean-field calculations with other force parameterizations [15] predict values between 0.28 and 0.31 . Hence, the properties of TFN can, in principle, test the predictive power of the different interactions used in HFB and relativistic mean-field calculations for nuclei far from 

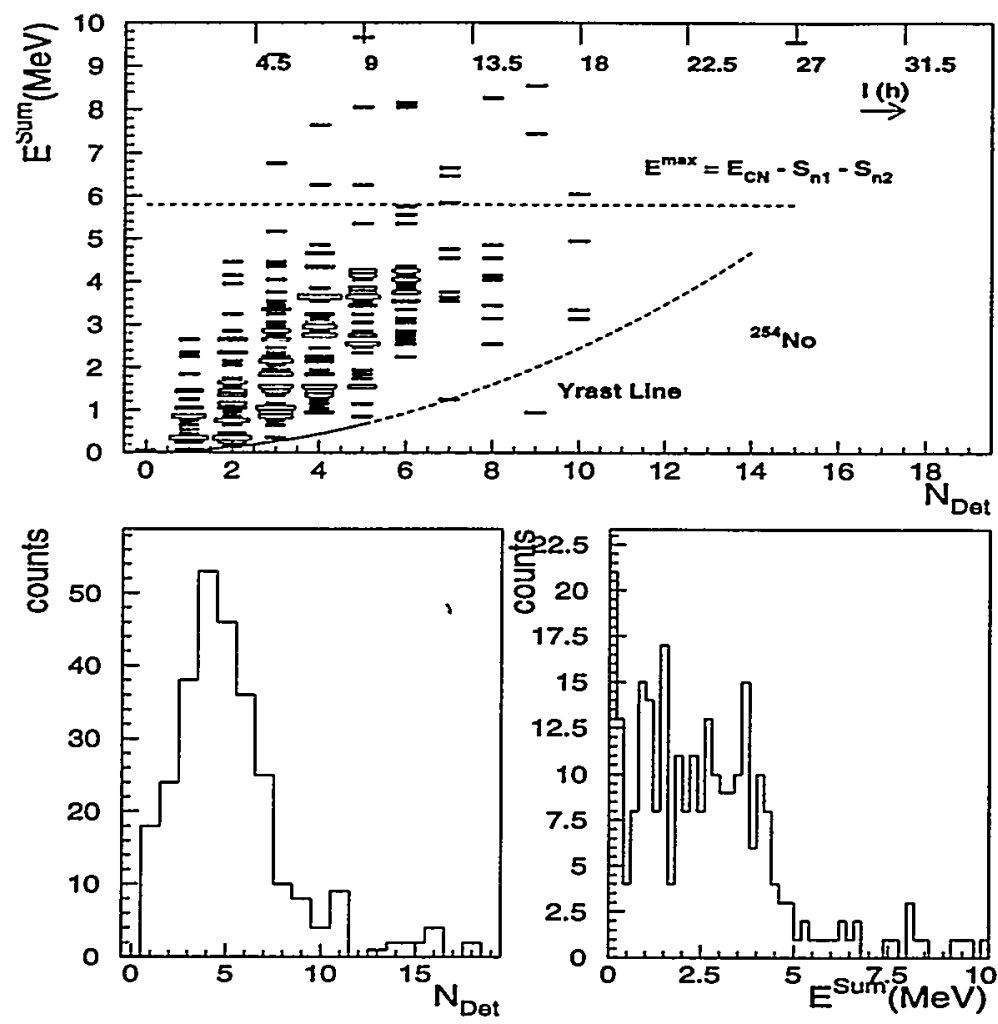

FIGURE 5. Two-dimensional spectrum of the Gammasphere module multiplicity vs. the sum-energy and the total projections. (A Gammasphere module is a combined detector unit consisting of a Ge-detector and the surrounding BGO shield.) The measured distribution is concentrated between the measured yrast line and the maximum excitation energy after neutron emission $\mathrm{E}^{m a x}=E_{C N}-S_{n 1}-S_{n 2}$.

stability.

The measured moments of inertia increase with spin, as seen in Fig. 3, probably due to the gradual alignment of quasiparticles, specifically those occupying the high- $j$ proton $i_{13 / 2}$ and neutron $j_{15 / 2}$ orbițals. Hence, the increase of $\mathcal{J}^{(2)}$ and $\mathcal{J}^{(1)}$ with frequency can provide a stringent test of theory. However, no calculations of finite-spin properties have been published so far.

The observation of states with spin up to 14 implies that neutron evaporation can compete against fission up to at least that spin. A fission barrier must still exist up to that angular momentum in ${ }^{254}$ No. Preliminary analysis of the multiplicity distribution from our experiment suggests that residues are formed with spin up to 18 . Our data further imply that the shell-correction energy, which creates the barrier, is reasonably robust with spin. They also demonstrate that high-spin states of TFN can be studied by means of (HI,xn) reactions. For future work, it will be interesting to investigate the fission barrier and its dependence on angular momentum to provide better insight into the production mechanism of the heaviest elements. 
We acknowledge the efforts of the ATLAS crew in providing the required currents of ${ }^{48} \mathrm{Ca}$ and of $\mathrm{B}$. Nardi in the operation of the target wheel. We are grateful for discussions with and private communications from P-H. Heenen, G. Lalazissis, A. Macchiavelli, W. Nazarewicz, K. Rutz, R. Smolanczuk and A. Sobiczewski. The work is supported by the U.S. Dept. of Energy, under Contract Nos. W-31-109ENG-38 and DE-AC03-76SF00098, the U.S. National Science Foundation, the U.K. Engineering and Sciences Research Council and a NATO grant (for S.S.) through the Research Council of Norway.

\section{REFERENCES}

1. P. Möller and J.R. Nix, J. Phys. G20, 1681 (1994).

2. R. Smolanczuk et al., Phys. Rev. C52, 1871 (1995).

3. S. Hofmann, Rep. Prog. Phys. 61, 639 (1998).

4. S. G. Nilsson et al., Nucl. Phys. A115, 545 (1968).

5. Z. Patyk and A. Sobiczewski, Nucl. Phys. A533, 132 (1991).

6. H.W. Gäggeler et al., Nucl. Phys. A502, 561c (1989).

7. J.E. Draper et al., Phys. Rev. C42, R1791 (1990); J. Becker et al., ibid. C46, 889 (1992); G. Hackman et al., Phys. Rev. Lett. 79, 4100 (1997).

8. L. Grodzins, Phys. Lett. 2, 88 (1962).

9. S. Raman et al., At. Data Nucl. Data Tables 42, 1 (1989).

10. W. Nazarewicz and I. Ragnarsson, Handbook Of Nuclear Properties, Clarendon Press, Oxford, 1996, ed. D. Poenaru and W. Greiner, p. 97.

11. S. Cwiok et al., Nucl. Phys. A573, 356 (1994).

12. P. Möller et al., At. Data Nucl. Data Tables 59, 185 (1995).

13. S. Cwiok et al., Nucl. Phys. A611, 211 (1996); W. Nazarewicz and P-H. Heenen, priv. comm. (1998).

14. G. Lalazissis et al., priv. comm. (1998); Nucl. Phys. A608, 202 (1996).

15. T. Bürvenich et al., Eur. Phys., in press; J. K. Rutz et al., Phys. Rev. C 56, 238 (1997). 